\title{
Intermittent-Contact Heterodyne Force Microscopy
}

\author{
M. Teresa Cuberes \\ Laboratory of Nanotechnology, University of Castilla-La Mancha, Plaza Manuel de Meca 1, 13400 Almadén, Spain
}

Correspondence should be addressed to M. Teresa Cuberes, teresa.cuberes@uclm.es

Received 9 December 2008; Accepted 26 January 2009

Recommended by Min Xu

\begin{abstract}
Heterodyne Force Microscopy opens up a way to monitor nanoscale events with high temporal sensitivity from the quasistatic cantilever mechanical-diode response taking advantage of the beat effect. Here, a novel heterodyne ultrasonic force method is proposed, in which the cantilever is driven in amplitude-modulation mode, at its fundamental flexural eigenmode. Ultrasonic vibration in the megahertz range is additionally input at the tip-sample contact from the cantilever base and from the back of the sample. The ultrasonic frequencies are chosen in such a way that their difference is coincident with the second cantilever eigenmode. In the presence of ultrasound, cantilever vibration at the difference frequency is detected. Similarly as in heterodyne force microscopy, it is expected that the phase response yields information with increased sensitivity due to the beat effect.
\end{abstract}

Copyright () 2009 M. Teresa Cuberes. This is an open access article distributed under the Creative Commons Attribution License, which permits unrestricted use, distribution, and reproduction in any medium, provided the original work is properly cited.

\section{Introduction}

The mechanical-diode (MD) approach is based on the detection of the quasistatic response of an Atomic Force Microscopy (AFM) cantilever when the forces actuating upon the tip vary nonlinearly in the ultrasonic time scale $[1,2]$. Up to now, the mechanical-diode response has been mostly exploited with the AFM working in contact or nearcontact mode [3, 4]. Heterodyne Force Microscopy (HFM) [5] introduced a novel method, in which the cantilever tip is in contact with the sample surface, and ultrasound is excited both at the tip (from a transducer at the cantilever base) and at the sample surface (from a transducer at the back of the sample) at adjacent frequencies, and mixed at the tip-sample gap. By this procedure, the tip-sample distance is modulated in beats, and extremely small phase shifts of the sample ultrasonic vibration can be easily monitored via the much larger phase shift of the cantilever vibration induced at the difference (beat) frequency (beat effect). Here, I propose a novel heterodyne ultrasonic force method, named hereafter Intermittent-Contact Heterodyne Force Microscopy (IC-HFM) in which the cantilever is driven in tapping mode, at its fundamental resonance. Ultrasonic vibration in the megahertz range is additionally input at the tip-sample contact from the cantilever base and from the back of the sample. The ultrasonic frequencies are so chosen that their difference is coincident with the second-order cantilever resonance. Here, I demonstrate that in the presence of ultrasound, cantilever vibration at the difference frequency is detected. As explained below, the results can be attributed to the activation of a mechanicaldiode signal during the time that the tip and the sample are in contact. The cantilever response can be controlled by varying the difference frequency, which is kept nearest to the second-order cantilever resonance. Phase data are expected to provide an increased sensitivity via the beat effect.

\section{Principle of Measurement: Intermittent-Contact Heterodyne Force Microscopy (IC-HFM)}

The principle of measurement in IC-HFM is illustrated in Figure 1. The fundamental resonance of the cantilever $\omega_{0}$ is excited using a piezoelement at the cantilever base. The AFM is operated in tapping mode, with the feedback keeping constant the amplitude of the fundamental flexural cantilever resonance. Additionally, ultrasonic vibration at frequencies $\omega_{1}$ and $\omega_{2}$ is input at the tip-sample contact from ultrasonic piezos located at the cantilever base and at the back of the sample, respectively. Frequencies $\omega_{1}$ and $\omega_{2}$ are chosen in such a way that $\left|\omega_{2}-\omega_{1}\right|$ equals the second-order cantilever resonance. As the cantilever is driven 


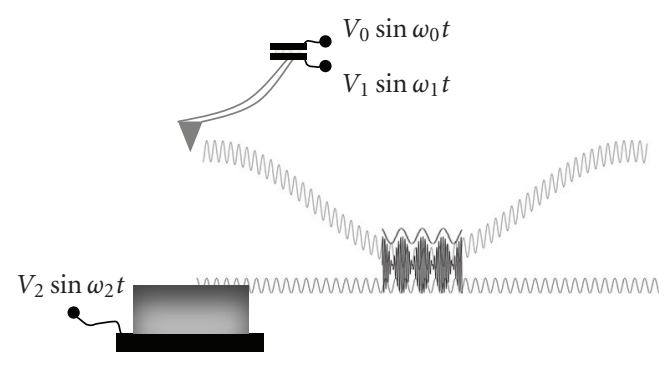

FIgUre 1: A schematic diagram illustrating IC-HFM (see text).

in its fundamental mode, it comes in contact with the sample surface for a certain time every period. During this contact time, the tip-sample distance is modulated in beats at the difference frequency $\left|\omega_{2}-\omega_{1}\right|$ due to the tip and sample ultrasonic vibration and, provided that the tip and sample ultrasonic vibration amplitudes are sufficiently large, a mechanical-diode force will act upon the cantilever and resonantly excite its second-order vibration mode. As in HFM [5], the phase cantilever response at the difference frequency $\left|\omega_{2}-\omega_{1}\right|$ is expected to provide information about tip-sample interactions with increased time sensitivity due to the beat effect.

\section{Experiment}

The measurements have been implemented by appropriately modifying a commercial AFM instrument (NANOTEC). The NANOTEC electronics was used for AFM operation in tapping mode, with the feedback keeping constant the amplitude of fundamental flexural cantilever resonance $\omega_{0}$. Ultrasonic piezoelements were additionally attached to the sample and the tip holders (see Figure 1). Function generators were used to simultaneously excite the sinusoidal vibration of the sample surface and the cantilever tip at two frequencies $\omega_{1}$ and $\omega_{2}$ in the megahertz range $(\approx 4 \mathrm{MHz})$. The difference frequency $\left|\omega_{2}-\omega_{1}\right|$ was purposely chosen to be coincident with the second-order cantilever resonance. Electronic mixing of the synchronous signals from both generators provided the reference signal for a lock-in amplifier to track the cantilever response at the difference frequency $\left|\omega_{2}-\omega_{1}\right|$ in amplitude and phase. Rectangular Si cantilevers were used: VISTA probes provided by Nanoscience Instruments, with nominal spring constant $3 \mathrm{~N} \mathrm{~m}^{-1}$, and resonance frequencies $\omega_{0} \approx 55 \mathrm{KHz}$ and $\omega_{\text {second_mode }} \approx 350 \mathrm{KHz}$. The samples consisted in titanium nitride ( $\mathrm{TiN}$ ) coatings prepared by dc magnetron sputtering onto polished AISI 304 stainless steel discs in a vacuum chamber at room temperature using a water-cooled Ti target [6].

\section{Results and Discussion}

Figures 2(a) and 2(b) show the fundamental and the secondfree flexural cantilever resonances, respectively. Figure 2(c) shows the spectral cantilever response measured with the FFT facility of our oscilloscope (Agilent DSO6104A) with

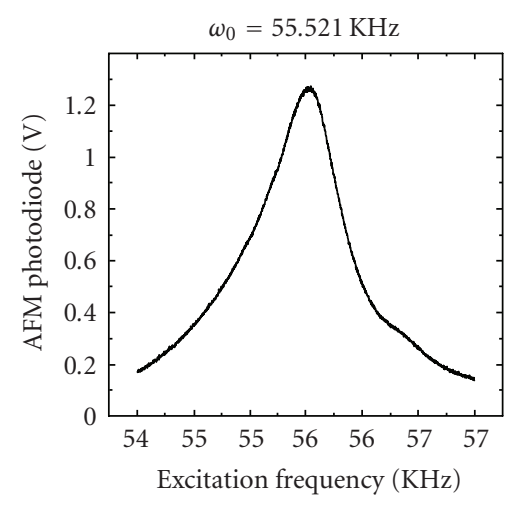

(a)

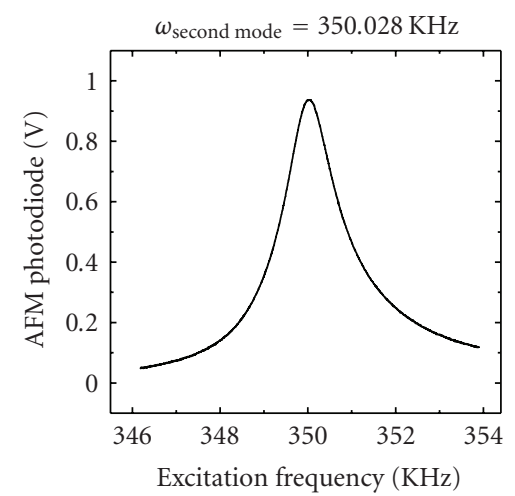

(b)

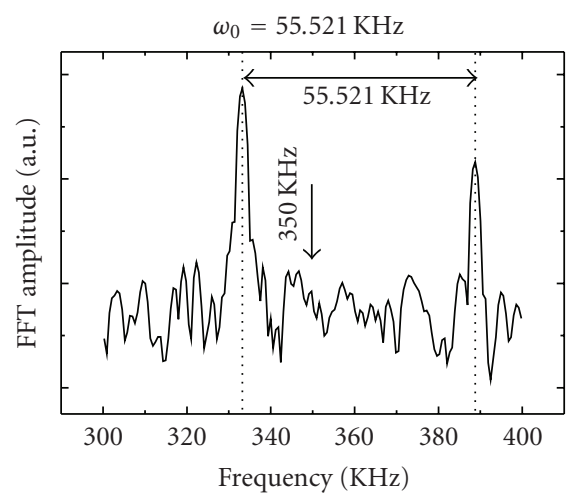

(c)

Figure 2: (a) Fundamental and (b) second-order flexural cantilever resonances measured with the cantilever tip out-of-contact with the sample surface (free cantilever modes), in the absence of ultrasonic vibration. (c) FFT of the AFM photodiode signal in tapping mode operation: excitation frequency $\omega_{0}=55.521 \mathrm{KHz}$, set-point amplitude $V=15 \% V_{0}$.

the AFM operated in tapping mode. Peaks at $333.65 \mathrm{KHz}$ and $388.55 \mathrm{KHz}$ are apparent, which correspond to the 6 th and 7 th harmonics of the fundamental cantilever resonance. As it is well known [7-12], in tapping mode operation, a certain amount of power is shifted to higher harmonics due to the distortion of the harmonic motion of the cantilever at the bottom of each tapping oscillation cycle. Typically, highamplitude harmonics always appear close to the resonance 


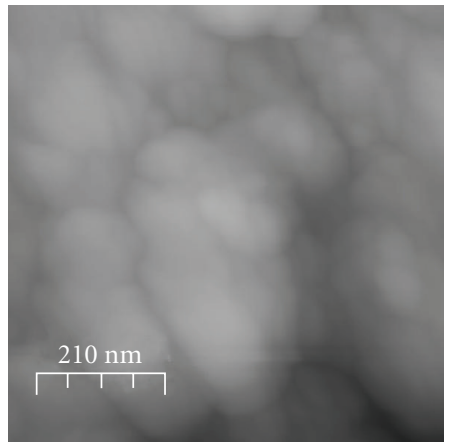

(a)

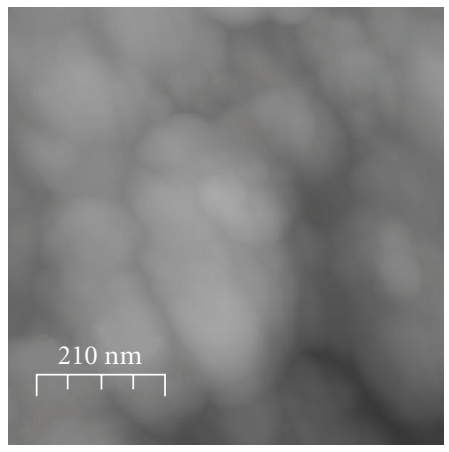

(b)

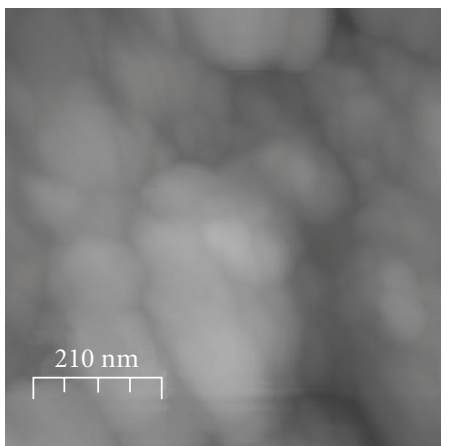

(c)

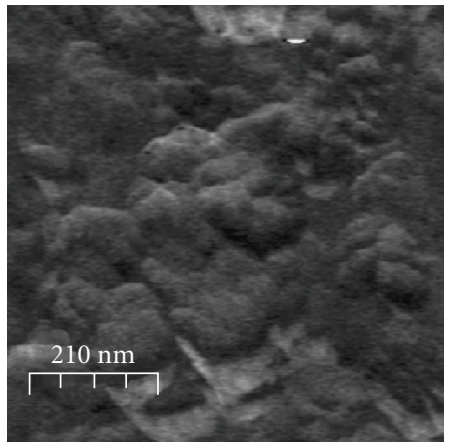

$\left(a^{\prime}\right)$

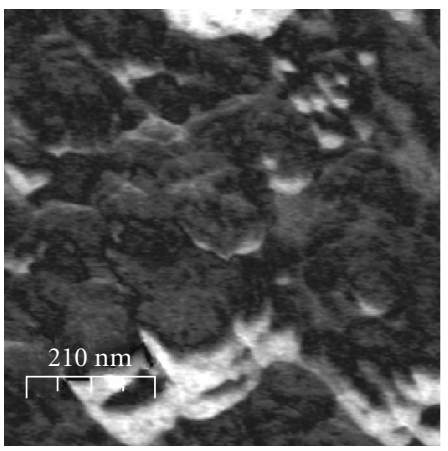

$\left(\mathrm{b}^{\prime}\right)$

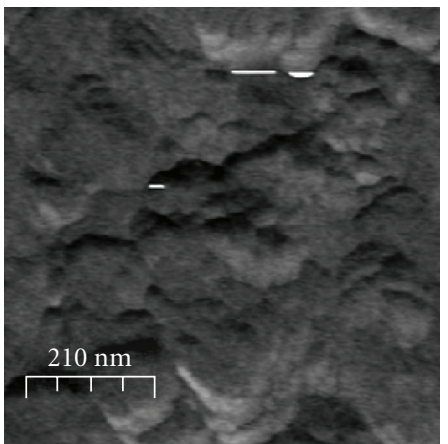

$\left(c^{\prime}\right)$

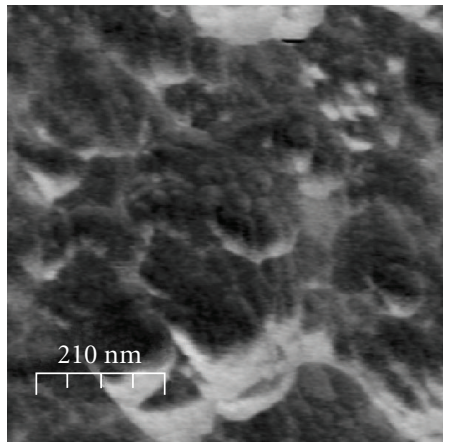

$\left(a^{\prime \prime}\right)$

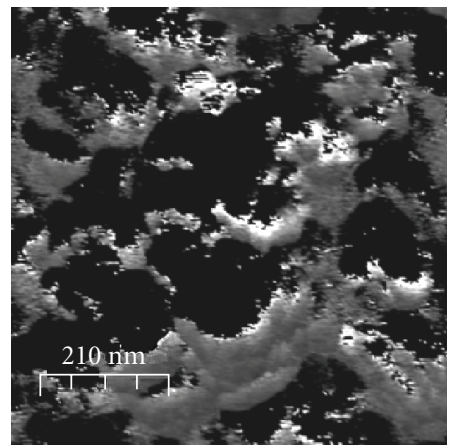

$\left(b^{\prime \prime}\right)$

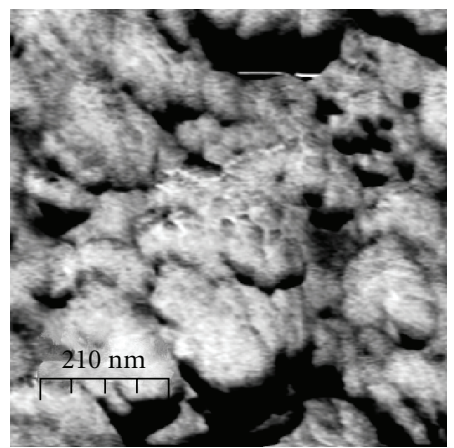

$\left(c^{\prime \prime}\right)$

Figure 3: Topography $(\mathrm{a})-(\mathrm{c})$ and IC-HFM amplitude $\left(\mathrm{a}^{\prime}\right)-\left(\mathrm{c}^{\prime}\right)$ and Phase $\left(\mathrm{a}^{\prime \prime}\right)-\left(\mathrm{c}^{\prime \prime}\right)$ images recorded over a same $(700 \times 700)$ nm ${ }^{2}$ surface region of a TiN coating. Images with the same letter (i.e., $(\mathrm{a}),\left(\mathrm{a}^{\prime}\right)$, and $\left.\left(\mathrm{a}^{\prime \prime}\right)\right)$ were simultaneously recorded. Tapping parameters: $\omega_{0}=$ $55.52 \mathrm{KHz}, V=31 \% V_{0}$. Ultrasonic parameters: (a)-(a") $\omega_{1}=4.800 \mathrm{MHz}, \omega_{2}=4.449 \mathrm{MHz}$; (b) $-\left(\mathrm{b}^{\prime \prime}\right) \omega_{1}=4.800 \mathrm{MHz}, \omega_{2}=4.450 \mathrm{MHz}$; (c) $-\left(\mathrm{c}^{\prime \prime}\right) \omega_{1}=4.800 \mathrm{MHz}, \omega_{2}=4.451 \mathrm{MHz} . V_{1}$ and $V_{2}$ were kept constant. Greyscale range: $(\mathrm{a})-(\mathrm{c}) 35 \mathrm{~nm} ;\left(\mathrm{a}^{\prime}\right) 3.6 \mathrm{~V},\left(\mathrm{~b}^{\prime}\right) 6.5 \mathrm{~V},\left(\mathrm{c}^{\prime}\right) 3.2 \mathrm{~V}$; $\left(\mathrm{a}^{\prime \prime}\right) 4.2 \mathrm{~V},\left(\mathrm{~b}^{\prime \prime}\right) 10 \mathrm{~V}$ (out-of-scale), ( $\left.\mathrm{c}^{\prime \prime}\right) 5.0 \mathrm{~V}$.

frequencies of higher cantilever resonant modes, indicating a dependence of harmonics generation on cantilever resonances. In our case, the frequency corresponding to the second-order cantilever resonant mode lies in between the 6th and 7th harmonics of the fundamental resonance, as indicated by the arrow in Figure 2(c). In the absence of ultrasound, cantilever vibration in its second-order resonant mode could not be observed from the FFT spectra (see Figure 2(c)). Nevertheless, in the presence of ultrasound, when ultrasonic signals of $\omega_{1}=4.800 \mathrm{MHz}$ and $\omega_{2} \approx$ $4.450 \mathrm{MHz}$ were simultaneously input to the cantilever tip and sample surfaces, respectively, while keeping the AFM in tapping mode operation (see Figure 1), a signal at $\mid \omega_{2}-$ $\omega_{1} \mid \approx 350 \mathrm{KHz}$ was clearly detected by means of the lock-in amplifier (see Figure 3). The lock-in used for the detection of the difference frequency was independent from the AFM electronics employed for tapping mode operation. The reference signal for the lock-in amplifier to detect cantilever vibration at the difference frequency of tip and sample ultrasonic vibrations was provided by electronically mixing the synchronous signals from the ultrasonic signal generators, using a simple mixer. A slight modification of the difference frequency led to significant variations in the signal detected by the lock-in amplifier. Figure 3 shows amplitude 
and phase outputs of the lock-in amplifier in different cases, together with the simultaneously recorded topographic image provided by the tapping-mode measurements. A maximum amplitude value of the signal detected by the lockin amplifier was obtained when the difference frequency of tip and sample ultrasonic vibrations was coincident with the second-order cantilever resonance mode (Figures 3(b)$3\left(b^{\prime \prime}\right)$, difference frequency of $\left.350 \mathrm{KHz}\right)$. Nevertheless, for this frequency, the phase response could not be properly measured. For difference frequency values slightly above or below the second cantilever resonance (Figures $3(\mathrm{a})-3\left(\mathrm{a}^{\prime \prime}\right)$, difference frequency of $349 \mathrm{KHz}$, and Figures $3(\mathrm{c})-3\left(\mathrm{c}^{\prime \prime}\right)$, difference frequency of $351 \mathrm{KHz}$, respectively), the phase contrast is reversed.

The excitation of cantilever vibration at the difference frequency of tip and surface ultrasonic vibrations is attributed to the activation of the mechanical-diode effect during the tip-sample contact time, as illustrated in Figure 1, in the same manner that it occurs in $\operatorname{HFM}[4,5]$. The procedures of Scanning Near Field Ultrasonic Holography (SNFM) [13] and Resonant Difference Frequency Atomic Force Ultrasonic [14] are similarly performed to HFM, with the difference (beat) frequency chosen in the range of hundreds of $\mathrm{KHz}$, above the first cantilever resonance frequency in [13], and coincident with a high-order cantilever contact resonance in [14]. Recently, it has been demonstrated that $\mathrm{GHz}$ vibrations from an acoustic resonator can be detected by operating the AFM in tapping mode using the amplitude of the fundamental cantilever mode to control the feedback and collect the topography, and the secondorder mode to detect acoustic information [15]. In ICHFM, the operation mode is actually the same as in [15], save that here the resonant excitation of the second-order cantilever mode is activated by mixing of surface and tip ultrasonic vibrations via the nonlinearity of the tip-surface interaction.

The simultaneous excitation of the first and second cantilever modes in amplitude modulation (AM) AFM operation is currently attracting a great deal of interest [1619]. It has been demonstrated that the sensitivity of the AMAFM to map compositional changes can be enhanced by the simultaneous excitation of the first and second flexural modes [18]. A theory has been developed that considers coupling of the different eigenmodes of the cantilever by the virial of the tip-surface forces, and explains the origin of the high force sensitivity observed in multifrequency force microscopy experiments [19]. Here, it is demonstrated that it is possible to detect and control cantilever vibration at frequencies near the second-order cantilever resonance by simultaneously exciting ultrasonic vibration at the tip and surface, while the AFM is driven in tapping mode. Tipsample interactions events that result in ultrasonic phase delays of surface versus tip vibration should be easily detected with high sensitivity taking into account the beat effect. The scope of the present work is limited to demonstrate the feasibility of the procedure, rather than to provide a detail understanding of the observed contrast. As it will be discussed elsewhere [20] TiN coatings may exhibit important differences in elastic contrast due to strain, structural defects, or coexistence of different phases. Contrast in ICHFM images in Figure 3 may originate because of elasticity inhomogeneities.

\section{Summary}

The results presented here demonstrate the detection of cantilever vibration at frequencies coincident or next to its second-order resonant mode, excited by ultrasonic vibration from the cantilever tip and from the sample surface mixed via the nonlinearity of the tip-surface contact, while the AFM is operated in tapping mode. The amplitude in the response is at a maximum when the difference frequency of surface and tip ultrasonic vibration is exactly coincident with the second-order cantilever resonance. Contrast in the phase images of the cantilever response is reversed depending on whether the resulting difference frequency is above or below the frequency of the second-order cantilever resonance.

\section{Acknowledgments}

The author thanks J. A. Hidalgo for assistance with the experiments. Financial support from the Junta de Comunidades de Castilla-La Mancha (JCCM) under Project PCI-08-092 is gratefully acknowledged.

\section{References}

[1] W. Rohrbeck and E. Chilla, "Detection of surface acoustic waves by scanning force microscopy," Physica Status Solidi A, vol. 131, no. 1, pp. 71-69, 1992.

[2] O. Kolosov and K. Yamanaka, "Nonlinear detection of ultrasonic vibrations in an atomic force microscope," Japanese Journal of Applied Physics, vol. 32, no. 8A, pp. L1095-L1098, 1993.

[3] M. T. Cuberes, "Nanoscale friction and ultrasonics," in Fundamentals of Friction and Wear on the Nanometer Scale, E. Gnecco and E. Meyer, Eds., pp. 49-71, Springer, Berlin, Germany, 2007.

[4] M. T. Cuberes, "Mechanical-diode mode ultrasonic friction force microscopies," in Applied Scanning Probe Methods, B. Bhushan, H. Fuchs, and H. Yamada, Eds., Springer, Berlin, Germany, 2009.

[5] M. T. Cuberes, H. E. Assender, G. A. D. Briggs, and O. V. Kolosov, "Heterodyne force microscopy of PMMA/rubber nanocomposites: nanomapping of viscoelastic response at ultrasonic frequencies," Journal of Physics D, vol. 33, no. 19, pp. 2347-2355, 2000.

[6] J. A. Hidalgo and C. M. Ocampo, "EIS and microstructural studies of TiN coatings deposited by PVD d.c. magnetron sputtering," ECS Transactions, vol. 6, no. 13, pp. 35-41, 2007.

[7] R. Hillenbrand, M. Stark, and R. Guckenberger, "Higherharmonics generation in tapping-mode atomic-force microscopy: insights into the tip-sample interaction," Applied Physics Letters, vol. 76, no. 23, pp. 3337-3483, 2000.

[8] R. W. Stark and W. M. Heckl, "Fourier transformed atomic force microscopy: tapping mode atomic force microscopy beyond the Hookian approximation," Surface Science, vol. 457, no. 1-2, pp. 219-228, 2000. 
[9] R. W. Stark and W. M. Heckl, "Higher harmonics imaging in tapping-mode atomic-force microscopy," Review of Scientific Instruments, vol. 74, no. 12, pp. 4989-5284, 2003.

[10] S. Crittenden, A. Raman, and R. Reifenberger, "Probing attractive forces at the nanoscale using higher-harmonic dynamic force microscopy," Physical Review B, vol. 72, no. 23, Article ID 235422, 13 pages, 2005.

[11] O. Sahin, C. F. Quate, O. Solgaard, and A. Atalar, "Resonant harmonic response in tapping-mode atomic force microscopy," Physical Review B, vol. 69, no. 16, Article ID 165416, 9 pages, 2004.

[12] J. Legleiter, M. Park, B. Cusick, and T. Kowalewski, "Scanning probe acceleration microscopy (SPAM) in fluids: mapping mechanical properties of surfaces at the nanoscale," Proceedings of the National Academy of Sciences of the United States of America, vol. 103, no. 13, pp. 4813-4818, 2006.

[13] G. S. Shekhawat and V. P. Dravid, "Nanoscale imaging of buried structures via scanning near-field ultrasound holography," Science, vol. 310, no. 5745, pp. 89-92, 2005.

[14] S. A. Cantrell, J. H. Cantrell, and P. T. Lillehei, "Nanoscale subsurface imaging via resonant difference-frequency atomic force ultrasonic microscopy," Journal of Applied Physics, vol. 101, no. 11, Article ID 114324, 8 pages, 2007.

[15] A. San Paulo, J. P. Black, R. M. White, and J. Bokor, "Detection of nanomechanical vibrations by dynamic force microscopy in higher cantilever eigenmodes," Applied Physics Letters, vol. 91, no. 5, Article ID 053116, 3 pages, 2007.

[16] T. R. Rodríguez and R. García, "Compositional mapping of surfaces in atomic force microscopy by excitation of the second normal mode of the microcantilever," Applied Physics Letters, vol. 84, no. 3, pp. 451-449, 2004.

[17] R. Proksch, "Multifrequency, repulsive-mode amplitudemodulated atomic force microscopy," Applied Physics Letters, vol. 89, no. 11, Article ID 113121, 3 pages, 2006.

[18] N. F. Martinez, S. Patil, J. R. Lozano, and R. Garcia, "Enhanced compositional sensitivity in atomic force microscopy by the excitation of the first two flexural modes," Applied Physics Letters, vol. 89, no. 15, Article ID 153115, 3 pages, 2006.

[19] J. R. Lozano and R. Garcia, "Theory of multifrequency atomic force microscopy," Physical Review Letters, vol. 100, no. 7, Article ID 076102, 4 pages, 2008.

[20] J. A. Hidalgo, C. Montero, and M. T. Cuberes, to be published. 

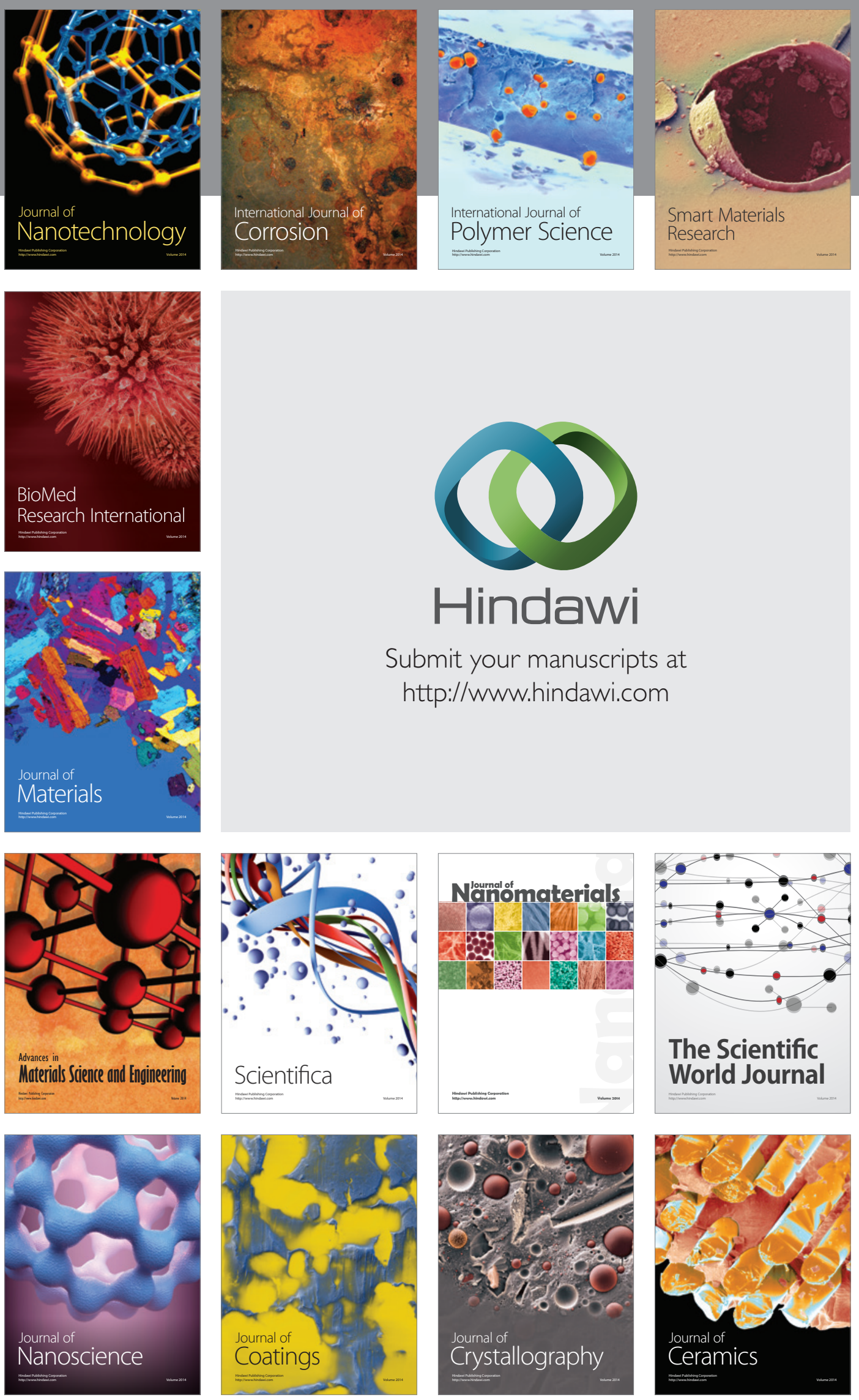

The Scientific World Journal

Submit your manuscripts at

http://www.hindawi.com

\section{World Journal}

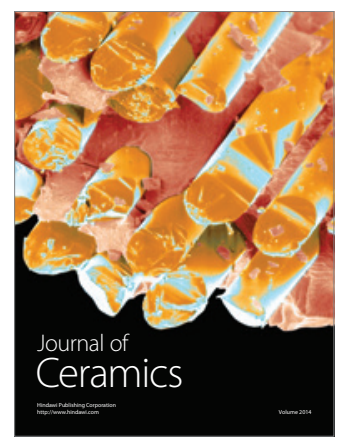

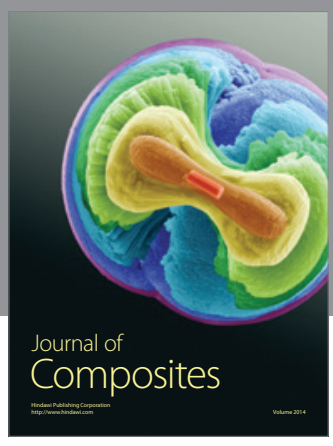
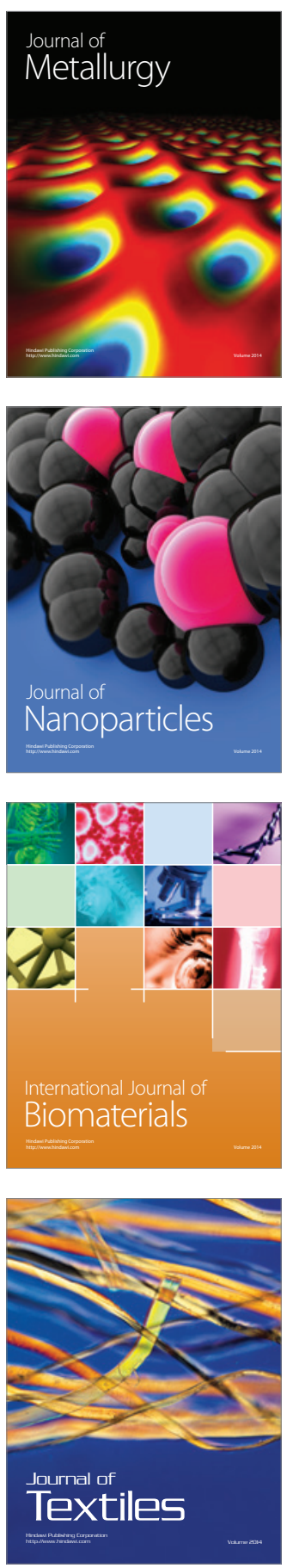